\title{
Stimulation of proteolytic activity of boar sperm acrosin by divalent metal ions
}

\author{
R. F. Parrish and K. L. Polakoski* \\ Departments of Urology and ${ }^{*}$ Obstetrics/Gynecology, Washington University School of \\ Medicine, 4960 Audubon Avenue, St Louis, Missouri 63110, U.S.A.
}

\begin{abstract}
Summary. The in-vitro proteolytic activity of boar sperm acrosin was stimulated by a series of monovalent and divalent metal ions. Equivalent concentrations of monovalent cations resulted in nearly identical increases in proteolytic activity, probably related to the increased ionic strength of the incubation medium. However, at concentrations of monovalent cations that resulted in a 2- to 3-fold stimulation of proteolysis of Azocoll, divalent metal ions caused a 24-(magnesium) to 46-(calcium) fold increase in proteolytic activity. We suggest that the divalent metal ion binds to acrosin and thus increases the proteolytic activity of acrosin to an extent greater than that due to the increased ionic strength of the incubation medium.
\end{abstract}

\section{Introduction}

Acrosin is presently thought to be one of the hydrolytic enzymes required for sperm penetration of the outer investments of the ovum (McRorie \& Williams, 1974). However, acrosin has also been postulated to play a role in the acrosome reaction (a membrane fusion and vesiculation that occurs between the outer acrosomal membrane and the plasma membrane, resulting in the loss of the acrosomal contents and exposing the inner acrosomal membrane (Bedford, 1968, 1970; Franklin, Barros \& Fussell, 1970)). This postulated role for acrosin in the acrosome reaction results from the observation that in the presence of the known acrosin inhibitors, benzamidine, $p$-aminobenzamidine, tosyl lysine chloromethyl ketone and $p$-nitrophenylguanidinobenzoate, the acrosome reaction of hamster spermatozoa was inhibited (Meizel \& Lui, 1976). If acrosin is directly involved in the membrane vesiculation reaction, as was postulated by Gordon (1973), acrosin would be digesting an insoluble membrane-bound protein. In addition, it is well documented that calcium is required for the acrosome reaction (Yanagimachi \& Usui, 1974; Summers, Talbot, Keough, Hylander \& Franklin, 1976; Talbot, Summers, Hylander, Keough \& Franklin, 1976). It seems reasonable that if acrosin is involved in the acrosome reaction, there might be a relationship between calcium concentration and the proteolytic activity of acrosin. The present experiments were therefore performed to determine if such a relationship did, in fact, exist.

\section{Materials and Methods}

Azocoll was purchased from Calbiochem-Behring Corp. Trypsin and $\mathrm{N}$ - $\alpha$-benzoyl-L-arginine ethyl ester (BzArgOEt) were purchased from Sigma. Denatured haemoglobin was a Worthington product. All other reagents were of the highest purity commercially available and were utilized without further purification. Glass-distilled, deionized water was used for the preparation of all buffers. 
Porcine $m_{\beta}$-acrosin (mol. wt 34000 ) was prepared from highly purified proacrosin that had been isolated from ejaculated boar spermatozoa as previously described (Polakoski \& Parrish, 1977; Parrish \& Polakoski, 1977).

Esterolytic activity of porcine acrosin was measured at $30^{\circ} \mathrm{C}$ by the method of Schwert \& Takenaka (1955). Acrosin $(0.9 \mu \mathrm{g})$ was added to a $3.0 \mathrm{ml}$ reaction volume consisting of 0.05 $\mathrm{M}$-Tris- $\mathrm{HCl}$, the appropriate cations at concentrations of $0.05 \mathrm{M}$ and $0.0005 \mathrm{M}-\mathrm{BzArgOEt}$. Hydrolysis of the substrate was measured spectrophotometrically at $253 \mathrm{~nm}$. A value of 1150 $\mathbf{M}^{-1} \mathrm{~cm}^{-1}$ was used to convert the change in absorbance to micromoles of BzArgOEt hydrolysed (Whitaker \& Bender, 1965).

Proteolytic activity of porcine acrosin against Azocoll was measured by the following procedure. Appropriate buffer solution $(1 \mathrm{ml}$ ) was placed in a plastic test tube containing $10 \mathrm{mg}$ Azocoll and the suspension was mixed by gentle vortexing. After equilibration for $10 \mathrm{~min}$ at $30^{\circ} \mathrm{C}$, an aliquot $(25 \mu \mathrm{l})$ of acrosin $(3.9 \mu \mathrm{g})$ was added. After an appropriate incubation period, the reaction was terminated by the addition of $200 \mu \mathrm{l} 0.2 \mathrm{M}$-benzamidine and the undigested Azocoll was removed by filtration through a glass wool-plugged Pasteur pipette. The absorbance at $520 \mathrm{~nm}$ was measured spectrophotometrically (Parrish \& Polakoski, 1977). Blanks were prepared and treated in the same manner except that $25 \mu \mathrm{l} 0.001 \mathrm{M}-\mathrm{HCl}$ were added instead of acrosin.

Proteolytic activity of porcine acrosin against haemoglobin was measured essentially by the method of Anson (1938). Denatured haemoglobin (2 g) was added to $98 \mathrm{ml}$ water, stirred for 15 min and clarified by centrifugation. To $0.5 \mathrm{ml}$ of the clarified haemoglobin solution was added $0.5 \mathrm{ml}$ of the appropriate buffer. The solution was incubated for $10 \mathrm{~min}$ at $30^{\circ} \mathrm{C}$ and the reaction initiated by the addition of $2.7 \mu \mathrm{g}$ acrosin. After incubation for $2 \mathrm{~h}$, the reaction was terminated by the addition of $0.62 \mathrm{ml} 5 \%$ trichloroacetic acid and after an additional $10 \mathrm{~min}$ at room temperature, the undigested haemoglobin was removed by filtration through a glass wool-plugged pipette. Blanks were prepared and treated in the same manner except that the acrosin was added after the trichloroacetic acid. Proteolysis was determined spectrophotometrically by measuring the absorbance at $280 \mathrm{~nm}$.

Exact buffer compositions and incubation times are given in the appropriate figure and table legends.

\section{Results}

Initial experiments to determine the effect of calcium on the proteolytic activity of acrosin were performed at $\mathrm{pH} 8.0$ in the presence or absence of $0.05 \mathrm{M}$-calcium chloride (Text-fig. 1). This concentration of calcium was selected since it had been previously established that the esterolytic activity of porcine acrosin was maximally stimulated (2-3-fold) by calcium at a concentration of $0.05 \mathrm{M}$ (Polakoski \& McRorie, 1973). In the presence of $0.05 \mathrm{M}$-calcium, there was a substantial increase in proteolytic activity (42-fold stimulation). To test whether calcium might have caused a conformational change in the Azocoll, resulting in the exposure of more arginine or lysine residues, the residues for which acrosin is specific (Polakoski \& McRorie, 1973), similar incubations of Azocoll in the presence and absence of calcium were performed with trypsin, a proteinase which, like acrosin, is highly specific for arginine- and lysine-containing residues (Walsh, 1970). There was no dramatic stimulation of the proteolysis of Azocoll by trypsin: activity was $1.17 \pm 0.09$ and $1.53 \pm 0.08$ at $520 \mathrm{~nm}$ (mean \pm s.d. for triplicate determinations of incubations at $\mathrm{pH} 8.0$ and for $25 \mathrm{~min}$ ).

Table 1 shows that a calcium concentration as low as $0.002 \mathrm{M}$ resulted in a doubled rate of proteolysis of Azocoll, relative to the rate of proteolysis in the absence of calcium. In addition, as the calcium concentration was increased, the rate of proteolysis increased. The effects of different concentrations of sodium chloride were compared to those of calcium chloride with 


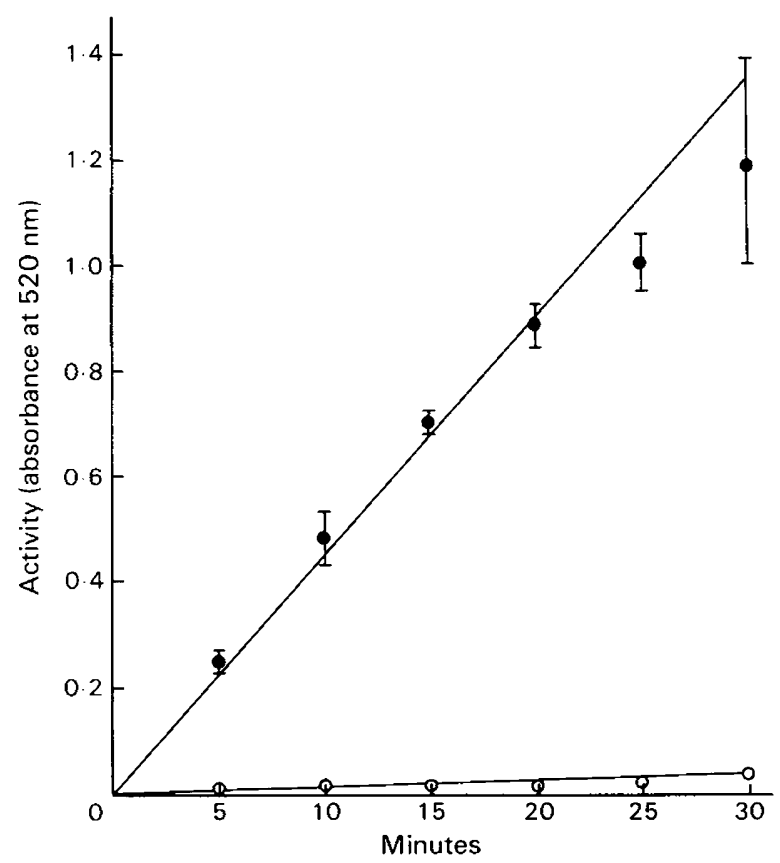

Text-fig. 1. Time course for the proteolytic activity of acrosin in the presence and absence of calcium. The reaction mixtures consisted of $0.05 \mathrm{M}$-Tris $-\mathrm{HCl}$ and either no calcium (O) or 0.05 $\mathrm{M}$-calcium chloride (O). Each value represents the mean \pm s.d. for 3 determinations. Error bars are not shown when they are less than the size of the symbol.

Table 1. The effect of calcium concentration on the acrosin-catalysed proteolysis of Azocoll

\begin{tabular}{ccc}
\hline $\begin{array}{c}\text { Calcium } \\
\text { conc. (mM) }\end{array}$ & $\begin{array}{c}\text { Activity } \\
\text { (absorbance at 520 nm) }\end{array}$ & Stimulation \\
\hline 0 & $0.021 \pm 0.003$ & - \\
2 & $0.056 \pm 0.006$ & $\times 2.7$ \\
5 & $0.108 \pm 0.001$ & $\times 5.1$ \\
10 & $0.176 \pm 0.018$ & $\times 8.4$ \\
15 & $0.278 \pm 0.012$ & $\times 13.2$ \\
25 & $0.454 \pm 0.006$ & $\times 21.6$ \\
50 & $0.981 \pm 0.024$ & $\times 46.7$ \\
\hline
\end{tabular}

The reaction mixtures at $\mathrm{pH} 8.0$ consisted of $0.05 \mathrm{M}$ Tris$\mathrm{HCl}$ and calcium chloride and were incubated for $25 \mathrm{~min}$. Activity values are expressed as the mean \pm s.d. for triplicate determinations and the degree of stimulation was calculated by dividing the activity in the presence of calcium by the activity in the absence of added calcium.

respect to proteolysis (Text-fig. 2). Although there was some stimulation of Azocoll digestion by increasing concentrations of sodium chloride, there was a much greater stimulation of the proteolysis by calcium, thus establishing that increased ionic strength was not the sole reason for the increased proteolysis observed in the presence of calcium.

Examination of the effect of a series of monovalent and divalent cations on the acrosin-catalysed proteolysis of Azocoll (Table 2) showed that calcium was not the exclusively effective divalent cation. Divalent metal ions were much more potent stimulators of acrosin-catalysed proteolysis than were the monovalent cations. 


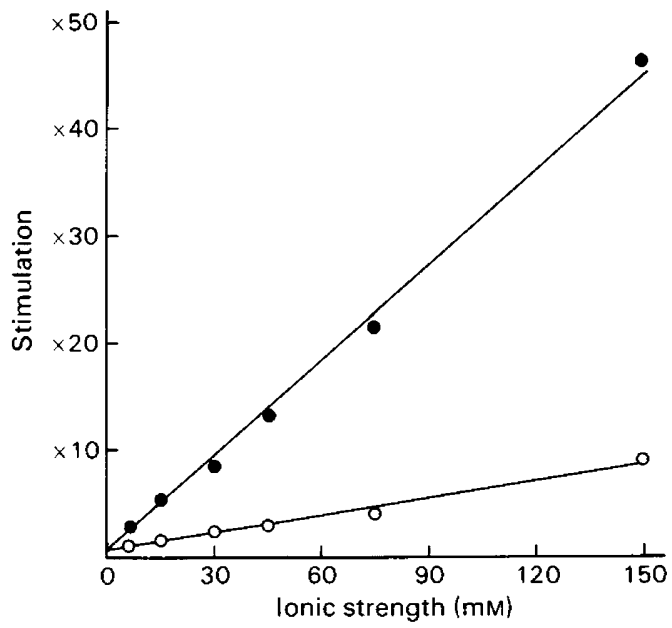

Text-fig. 2. The effect of ionic strength on the calcium stimulation of the proteolytic activity of acrosin. The reaction mixtures at $\mathrm{pH} 8.0$ consisted of $0.05 \mathrm{M}$-Tris- $\mathrm{HCl}$ and either calcium chloride (O) or sodium chloride $(0)$ at the indicated ionic strengths. The incubation period was $25 \mathrm{~min}$. Activity at each salt concentration was measured in triplicate and the degree of stimulation was calculated by dividing the activity in the presence of added salt by the activity in the absence of added salt.

Table 2. The effect of monovalent and divalent cations on (a) the acrosin-catalysed hydrolysis of Azocoll, (b) the proteolysis of haemoglobin, and (c) the esterolytic activity of acrosin

\begin{tabular}{|c|c|c|c|c|c|c|}
\hline \multirow[b]{2}{*}{ Cation added } & \multicolumn{2}{|c|}{ Azocoll } & \multicolumn{2}{|c|}{ Haemoglobin } & \multicolumn{2}{|c|}{ BzArgOEt } \\
\hline & $\begin{array}{c}\text { Activity } \\
\text { (absorbance } \\
\text { at } 520 \mathrm{~nm} \text { ) }\end{array}$ & Stimulation & $\begin{array}{c}\text { Activity } \\
\text { (absorbance } \\
\text { at } 280 \mathrm{~nm} \text { ) }\end{array}$ & Stimulation & $\begin{array}{c}\text { Activity } \\
(\mu \mathrm{mol} / \mathrm{min}) \\
\times 10^{2}\end{array}$ & Stimulation \\
\hline None & $0.029 \pm 0.006$ & - & $0.055 \pm 0.018$ & - & $6.7 \pm 0.40$ & - \\
\hline Ammonium & $0.080 \pm 0.005$ & $\times 2.8$ & $0.078 \pm 0.014$ & $\times 1.4$ & $13.2 \pm 0.92$ & $\times 1.97$ \\
\hline Lithium & $0.069 \pm 0.003$ & $\times 2.4$ & $0.061 \pm 0.005$ & $\times 1 \cdot 1$ & $10.8 \pm 0.18$ & $\times 1.61$ \\
\hline Potassium & $0.079 \pm 0.005$ & $\times 2.7$ & $0.070 \pm 0.012$ & $\times 1 \cdot 3$ & $12.0 \pm 0.45$ & $\times 1.79$ \\
\hline Sodium & $0.069 \pm 0.008$ & $\times 2.4$ & $0.065 \pm 0.012$ & $\times 1.2$ & $11.7 \pm 0.78$ & $\times 1.74$ \\
\hline Barium & $1 \cdot 160 \pm 0.050$ & $\times 40$ & $0.419 \pm 0.033$ & $\times 7.6$ & $23.2 \pm 0.26$ & $\times 3.46$ \\
\hline Calcium & $1.340 \pm 0.082$ & $\times 46$ & $0.433 \pm 0.020$ & $\times 7.8$ & $23.1 \pm 0.54$ & $\times 3.45$ \\
\hline Magnesium & $0.700 \pm 0.021$ & $\times 24$ & $0.480 \pm 0.016$ & $\times 8.7$ & $23.4 \pm 0.15$ & $\times 3.49$ \\
\hline Strontium & $1.140 \pm 0.056$ & $\times 39$ & $0.455 \pm 0.056$ & $\times 8.3$ & $23.7 \pm 0.52$ & $\times 3.54$ \\
\hline
\end{tabular}

The reaction mixtures at $\mathrm{pH} 8.0$ consisted of $0.05 \mathrm{M}$-Tris- $\mathrm{HCl}$ (plus $0.005 \mathrm{M}$-BzArgOEt in (c)) and the indicated cations ( $0.005 \mathrm{M}$ ) added as the chloride salts. The incubation times were (a) $25 \mathrm{~min}$, (b) $2 \mathrm{~h}$ and (c) $25 \mathrm{~min}$.

Activity values are expressed as the mean \pm s.d. for triplicate determinations and the degree of stimulation was calculated by dividing the activity in the presence of added cation by the activity in the absence of added cation.

Because Azocoll is an insoluble proteinase substrate, it was important to determine if the divalent metal ion stimulation of proteolysis was restricted to Azocoll, or if stimulation of proteolysis would occur when a soluble substrate was utilized. The presence of monovalent cations in the incubation medium resulted in a slight, uniform increase in the proteolysis of haemoglobin relative to the proteolysis in the absence of added monovalent cations (Table 2), but a substantially higher degree of proteolysis was observed when the incubation mixture contained divalent metal ions. All the divalent metal ions tested gave approximately equal stimulation of proteolysis of haemoglobin but the stimulation was not as great as that when Azocoll was the substrate. All the divalent metal ions tested stimulated the esterolytic activity of 
acrosin to approximately the same degree, which was greater than that caused by monovalent cations. The effect of divalent metal ions on esterolytic activity was less than that on proteolytic activity against haemoglobin and substantially less than that on proteolysis of haemoglobin.

The acrosin-catalysed proteolysis of Azocoll was stimulated by calcium at $\mathrm{pH} 5 \cdot 0-8.0$ but the effect was greater at $\mathrm{pH} 7.0$ and 8.0 than at $\mathrm{pH} 5.0$ and 6.0 (Table 3).

Table 3. The effect of $\mathrm{pH}$ on the calcium stimulation of the acrosin-catalysed proteolysis of Azocoll

\begin{tabular}{|c|c|c|c|}
\hline $\mathrm{pH}$ & Calcium & $\begin{array}{c}\text { Activity } \\
\text { (absorbance at } 520 \mathrm{~nm} \text { ) }\end{array}$ & Stimulation \\
\hline \multirow[t]{2}{*}{8.0} & - & $0.020 \pm 0.001$ & \\
\hline & + & $0.977 \pm 0.078$ & $\times 49$ \\
\hline \multirow[t]{2}{*}{$7 \cdot 0$} & - & $0.024 \pm 0.002$ & \\
\hline & + & $0.963 \pm 0.047$ & $\times 40$ \\
\hline \multirow[t]{2}{*}{$6 \cdot 0$} & - & $0.037 \pm 0.003$ & \\
\hline & + & $0.681 \pm 0.007$ & $\times 18$ \\
\hline \multirow[t]{2}{*}{$5 \cdot 0$} & - & $0.061 \pm 0.004$ & \\
\hline & + & $0.523 \pm 0.016$ & $\times 8.5$ \\
\hline
\end{tabular}

The buffers and incubation times were: $\mathrm{pH} 8.0$, Tris, $25 \mathrm{~min} ; \mathrm{pH} 7 \cdot 0$, HEPES ( $N$-2-hydroxyethylpiperazine- $N^{\prime}-2$ ethanesulphonic acid), $90 \mathrm{~min} ; \mathrm{pH} 6.0$, MES (2-[N-

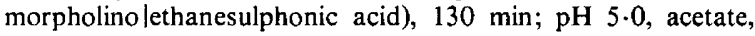
$170 \mathrm{~min}$. Activity values are expressed as the mean \pm s.d. for triplicate measurements and the degree of stimulation was calculated by dividing the activity in the presence of calcium by the activity in the absence of calcium.

\section{Discussion}

The present results demonstrate that the proteolytic activity of boar sperm acrosin is dramatically stimulated by divalent metal ions in vitro. Two reasonable explanations for this effect may be postulated. First, proteolysis can be stimulated by increases in the ionic strength of the incubation medium, as shown in Text-fig. 2 and Table 2. However, at concentrations of calcium that resulted in a 46-fold increase in the proteolysis of Azocoll, equivalent concentrations of monovalent cations resulted in only a 2 - to 3-fold stimulation of proteolysis. Qualitatively similar results were observed with both the low molecular weight substrate BzArgOEt, and the soluble proteinase substrate, haemoglobin. Therefore, increased ionic strength cannot explain the stimulation observed with divalent metal ions and a second hypothesis is required. As shown in Table 2, the increased proteolytic activity of acrosin in the presence of calcium and other divalent metal ions appears to correlate with the presence of a divalent metal ion-binding site on acrosin, which results in a stimulation of proteolytic activity greater than that which can be accounted for simply by an increase in the ionic strength. Of the metal ions tested, calcium appears to be one of the best stimulators of proteolysis. Zinc, which has been reported to be an inhibitor of acrosin (Meizel \& Mukerji, 1976), was not included in these experiments because under the experimental conditions used the zinc precipitated.

Although these in-vitro results clearly show that divalent metal ions stimulated the proteolytic activity of acrosin, the physiological role for the divalent metal ion-stimulation of acrosin proteolytic activity cannot be unequivocally delineated. There are, however, several possibilities. Magnesium can inhibit the calcium-dependent acrosome reaction (Rogers \& Yanagimachi, 1976) and strontium can replace calcium as a stimulator of the acrosome reaction (Yanagimachi \& Usui, 1974). Since both magnesium and strontium stimulated the proteolytic activity of acrosin (Table 2), this suggests that acrosin was not the locus of inhibition of the acrosome reaction by magnesium. 
Srivastava, Akruk \& Williams (1979) reported that extracts of rabbit spermatozoa contained hydrolytic enzymes, probably non-acrosin proteinases, that were required for digestion of the zona pellucida of the ovum. These results were based on the observations that in the presence of extracts of rabbit spermatozoa: (1) calcium stimulated total esterolytic activity against BzArgOEt; (2) calcium decreased the total proteinase activity as determined by Azocoll digestion; and (3) calcium inhibited the dissolution of the zona pellucida of rabbit ova. Although there may be differences between species, our results show that acrosin proteolytic activity was stimulated by calcium, and provide corollary evidence for the proposal by Srivastava et al. (1979) that hydrolyases in addition to acrosin are required for zona pellucida dissolution.

Finally, these results indicate that if calcium concentrations do increase in the acrosome before the vesiculation of the outer plasma membrane and the inner acrosomal membrane, then sufficient calcium concentrations might develop to stimulate the proteolytic activity of acrosin. Thus, while adding no additional evidence for a role for acrosin in the acrosome reaction, the present results are compatible with such a hypothesis.

This research was supported by National Institutes of Health Grants HD 09422 and 00296. We thank Dr Billy Day for generously donating the boar semen.

\section{References}

Anson, M.L. (1938) The estimation of pepsin, trypsin, papain and cathepsin with hemoglobin. $J$. gen. Physiol. 22, 79-89.

Bedford, J.M. (1968) Ultrastructural changes in the sperm head during fertilization in the rabbit. $A m . J$. Anat. 123, 329-357.

Bedford, J.M. (1970) Sperm capacitation and fertilization in mammals. Biol. Reprod., Suppl. 2, 128-158.

Franklin, L.E, Barros, C. \& Fussell, E.N. (1970) The acrosomal region and the acrosome reaction in sperm of the golden hamster. Biol. Reprod. 3, $180-200$.

Gordon, M. (1973) Localization of phosphatase activity on the membranes of the mammalian sperm head. $J$. exp. Zool. 185, 111-119.

McRorie, R.A. \& Williams, W.L. (1974) Biochemistry of mammalian fertilization. Ann. Rev. Biochem. 43, $777-803$.

Meizel, S. \& Lui, C.W. (1976) Evidence for the role of a trypsin-like enzyme in the hamster sperm acrosome reaction. J. exp. Zool. 195, 137-144.

Meizel, S. \& Mukerji, S.K. (1976) Biochemical studies of proacrosin and acrosin from hamster cauda epididymal spermatozoa. Biol. Reprod. 14, 444-450.

Parrish, R.F. \& Polakoski, K.L. (1977) Effect of polyamines on the activity of acrosin and the activation of proacrosin. Biol. Reprod. 17, 417-422.

Polakoski, K.L. \& McRorie, R.A. (1973) Boar acrosin. II. Classification, inhibition and specificity studies of a proteinase from sperm acrosomes. J. biol. Chem. 248, 8183-8188.
Polakoski, K.L. \& Parrish, R.F. (1977) Boar proacrosin purification and preliminary activation studies of proacrosin isolated from ejaculated boar sperm. $J$. biol. Chem. 252, 1888-1894.

Rogers, B.J. \& Yanagimachi, R. (1976) Competitive effect of magnesium on the calcium-dependent acrosome reaction in guinea pig spermatozoa. Biol. Reprod. 15, 614-619.

Schwert, G.W. \& Takenaka, Y. (1955) A spectro photometric determination of trypsin and chymotrypsin. Biochim. Biophys. Acta 16, 570-575.

Srivastava, P.N., Akruk, S.R. \& Williams, W.L. (1979) Dissolution of rabbit zona by sperm acrosomal extract: effect of calcium (1). J. exp. Zool. 207, $521-529$.

Summers, R.G., Talbot, P., Keough, E.M., Hylander, B.L. \& Franklin, L.E. (1976) Ionophore A23187 induces acrosome reactions in sea urchin and guinea pig spermatozoa (1). J. exp. Zool. 196, 381-385.

Talbot, P., Summers, R.G., Hylander, B.L., Keough, E.M. \& Franklin, L.E. (1976) The role of calcium in the acrosome reaction: an analysis using ionophore A23187.J. exp. Zool. 198, 383-391.

Walsh, K.A. (1970) Trypsinogens and trypsins of various species. Methods Enzymol. 19, 41-63.

Whitaker, J.R. \& Bender, M.L. (1965) Kinetics of papain-catalyzed hydrolysis of $a-N$-benzoyl- $L$ arginine ethyl ester and $\alpha-\mathrm{N}$-benzoyl-L-arginamide. $J$. Am. Chem. Soc. 87, 2728-2737.

Yanagimachi, R. \& Usui, N. (1974) Calcium dependence of the acrosome reaction and activation of guinea pig spermatozoa. Expl Cell Res. 89, 161-174. 